Testosteron und Tumorrisiko

\section{Frühe Glatze schützt vor Prostatakrebs}

- Wer einen hohen Testosteronspiegel hat, muss der Gefahr ins Auge sehen, dass ihm schon in jungen Jahren das Haupthaar abhanden kommt. Eine frohe Botschaft dazu verkündet indes eine US-amerikanische Studie, die kürzlich im Fachmagazin Cancer Epidemiology veröffentlicht wurde: Demzufolge erkranken Männer, bei denen sich die Glatze bereits im Alter von 30 Jahren ankündigt, später deutliche seltener an Prostatakrebs. Die Wissenschaftler begutachteten die Haarpracht von 999 Prostatakarzinom-Patienten und verglichen sie mit der von 942 gleichaltrigen Kontrollpersonen. Ausschlaggebend war dabei jedoch nicht der Zeitpunkt der Untersuchung, sondern der Kopfbewuchs im Alter von 30 Jahren. Zu diesem Zeitpunkt lichteten sich Schläfen und Hinterhaupt bei rund jedem vierten der Kontrollprobanden (25,2\%), aber nur bei jedem fünften der Karzinompatienten (19,8\%). Das entspricht einer Risikoreduktion von $29 \%$ im Zusammenhang mit testosteronbedingtem Haarausfall, folgern die Wissenschaftler. Je älter die Patienten zum

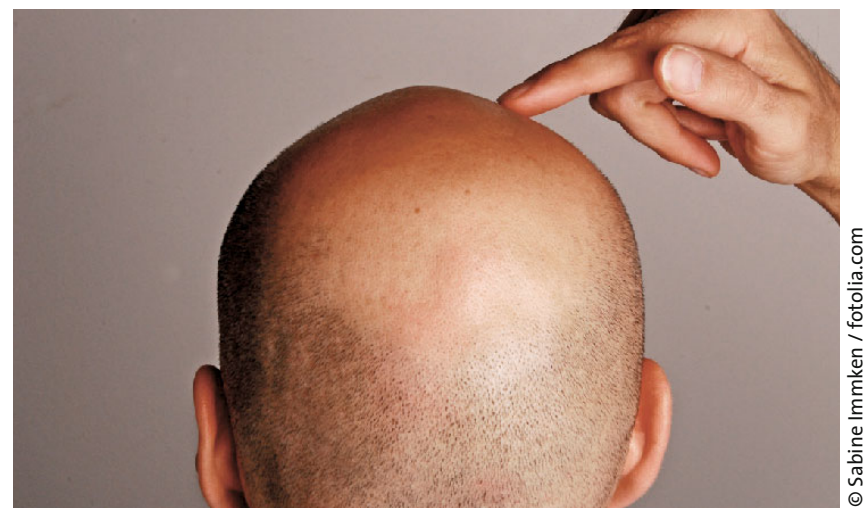

Männer mit Glatze können aufatmen. Denn wer früh die Haare verliert, hat einer Studie zufolge ein geringeres Risiko, ein Prostatakarzinom zu entwickeln.

Zeitpunkt der Untersuchung waren, desto deutlicher zeigte sich dieser Unterschied. Die Studienautoren postulieren daher, dass hohe Testosteronspiegel in jungen Jahren im Alter vor Prostatakrebs schützen können - allerdings bestehe auf diesem Gebiet noch weiterer Forschungsbedarf.

\section{DGU-Initiative zum Klinefelter-Syndrom}

\section{Interdisziplinäres Handeln gefragt}

— Die Deutsche Gesellschaft für Urologie (DGU) will besonders Eltern und Kinderärzte sensibilisieren, um eine frühe Behandlung von Jungen mit Klinefelter-Syndrom zu ermöglichen und Spätfolgen zu lindern. Zwar hat jeder 500. Mann ein zusätzliches X-Chromosom, erkannt wird die Abweichung der Geschlechtschromosomen aber nur in rund $20 \%$ der Fälle. Obwohl zu kleine Hoden spätestens bei der Musterung auffallen müssten, würde das Klinefelter-Syndrom zu oft erst erkannt, wenn die Männer wegen Unfruchtbarkeit behandelt werden.

„Aufgeklärte Eltern und Kinderärzte, aber auch Hausärzte und Internisten, die die J1-Untersuchung im Rahmen der kassenärztlichen Leistung im Alter zwischen zwölf und 14 Jahren vornehmen, können dazu beitragen, Träger des Klinefelter-Syndroms häufiger zu identifizieren“, betont DGU-Pressesprecherin Prof. Sabine Kliesch. Für den größtmöglichen Erfolg einer Therapie sei es wichtig, das Zeitfenster zwischen Pubertätsbeginn und frühem Erwachsenenalter, und zwar vor der dauerhaften Testosterongabe, für die Spermiengewinnung zu nutzen und das hänge entscheidend von der interdisziplinären Zusammenarbeit von Kinderärzten, Urologen, Endokrinologen und Reproduktionsmedizinern ab.

Zukünftig sollen individualisierte Therapien die Versorgung der Patienten weiter verbessern, so Zitzmann, dessen Münsteraner Team entsprechende interdisziplinäre Forschungen betreibt. „Wir erforschen zum Beispiel, ob in Zukunft tatsächlich alle Patienten Testosteron brauchen und wer aufgrund seiner genetischen Disposition vorbeugend auf bestimmte Folgeerkrankungen behandelt werden könnte", sagte der Androloge.

\section{Studien nach Arzneizulassung Krebsgesellschaft will Kassen mit ins Boot holen}

_ Krankenkassen sollten sich finanziell an wissenschaftlichen Studien zu bereits zugelassenen Arzneimitteln beteiligen, fordert der Generalsekretär der Deutschen Krebsgesellschaft Dr. Johannes Bruns. Im Gegenzug müsste die pharmazeutische Industrie den Kassen einen Rabatt auf den Arzneimittelpreis einräumen, so Bruns auf der MCCFachkonferenz „Onkologie 2010“ in Köln. Das könnte in die Preisverhandlungen zwischen Kassen und Pharmaindustrie eingebaut werden. „Über solche Rabattstudien können wir das Preisdumping und die Nutzensteigerung zusammenbringen." Die Krankenkassen würden in eine langfristig effizientere Nutzung der Arzneimittel investieren.

Bei der häufig diskutierten Diskrepanz zwischen den Kosten von Arzneimitteln und dem nachgewiesenen Nutzen dürfe das Interesse nicht nur darauf zielen, den Preis zu senken, forderte er. „Ich muss auch sehen, wie ich den Nutzen steigern kann, und das geht nur über Studien."

An vielen Studien, die von wissenschaftlichem Interesse sind, hätte die pharmazeutische Industrie nach der Zulassung kein Interesse. Man könne aber von den Kassen nicht verlangen, die Studien allein zu finanzieren. Für ein Engagement der Kassen müsste zunächst die gesetzliche Grundlage geschaffen werden. Dreiseitige Verträge zwischen Kassen, Herstellern und Wissenschaftlern könnten laut Bruns dazu beitragen, dass es in Deutschland mehr Untersuchungen zum direkten Vergleich zwischen zwei Produkten gibt. iss 